\title{
El aprendizaje desde la práctica: integración de nuevas metodologías docentes en Documentación
}

\author{
Fernanda Peset Mancebo \\ Antonia Ferrer Sapena \\ Nuria Lloret Romero \\ Universidad Politécnica de Valencia (España)
}

\subsection{Resumen}

Informe de una experiencia piloto de innovación docente pluridisciplinar realizada en la Universidad Politécnica de Valencia que consistió en integrar las actividades de formación de estudiantes de Documentación y Bellas Artes entorno a la realización de un documental sobre artistas, instituciones u organizaciones relacionadas con el Arte Contemporáneo valenciano, con el objetivo de luego emitirlo por la Televisión de la Universidad Politécnica. Esta metodología se acerca a la realidad del mercado laboral, en el que se suele trabajar en grupos de perfiles profesionales heterogéneos. Por otra parte, el estudiante se ejercita en la resolución de problemas informativos para un objetivo concreto, con unos plazos y usuarios que van a evaluar inmediatamente el resultado de su trabajo, interiorizando de forma real muchos contenidos aprendidos tan solo en teoría. En último lugar, el informe final ayuda al estudiante a sistematizar y verbalizar sus resultados y procesos de aprendizaje.

Palabras clave: Nuevas metodologías docentes. Integración de conocimientos. Educación y formación. Profesionales de la información.

\subsection{Abstract}

Report of a pilot experience of educational innovation in the Polytechnical University of Valencia (Spain). It consisted of integrating the work of various groups of students of Documentation and Art in a joined activity: producing documentaries on artists, institutions or organizations related to Valencian Contemporary Art, with the objective of emitting it through the University Television Broadcasting. This methodology is very near to the reality of the labour market, in which groups of professionals of heterogeneous profiles usually work together. On the other hand, the students work on the resolution of real and 
specific informative problems, with users who are going to evaluate the result of their work immediately. Last, the requisite of producing a final report helped the students to systematize their learning processes and results.

Keywords: New teaching methodologies. Knowledge integration in learning. Education and training. Information professionals.

\section{Introducción}

Los cambios en la sociedad española hacen de los estudios en documentación una pieza clave para el desarrollo de la sociedad de la información. Según las directrices de nuestro gobierno, los estudios universitarios se sustentan en tres pilares básicos (MECD, 2003): la sociedad del conocimiento, el aprendizaje continuo y la transmisión de la información a escala mundial. En este sentido, las declaraciones de La Sorbona (1998) y Bolonia (1999), el comunicado de Praga (2001) - revisado en Berlín-, y las acciones legislativas en nuestro país (LOU 2001) son el resultado de un proceso de convergencia que intenta adaptarse a este nuevo contex to europeo. Sus objetivos son el fomento de la calidad de la enseñanza, la armonización de los sistemas educativos y el desarrollo de metodologías educativas comparables a escala europea.

En España, según el MECD (2003, p. 9-10), esta situación de cambio ofrece una oportunidad única para establecer políticas coordinadas a escala europea y para experimentar en titulaciones piloto, estructuradas en redes nacionales financiadas por la propia UE, como ha ocurrido en Administración de Empresas de la Universidad de Salamanca, Matemáticas de la Universidad Autónoma de Madrid y de la Universidad de Cantabria y otras (Bayod y otros, 2002) - . El fin último - fomentar la "formación integral de los estudiantes, según las necesidades de la sociedad y de un mercado de trabajo cada vez más competitivo y sin fronteras" para Corcuera y Pagani (2002) - se está compendiando en una serie de iniciativas: modificación de los planes de evaluación y mejora, e instauración de un sistema de acreditaciones del profesorado; fomento la movilidad de profesorado y estudiantes; completa reestructuración de los estudios, armonizando los ciclos en la Unión Europea; inclusión de un suplemento al título que permita conocer las materias cursadas y la orientación de la titulación; homogenización de documentos - como guías docentes - e indicadores de calidad; implantación del sistema de créditos ECTS (European Credit Transfer System) que haga comparables las enseñanzas y evaluaciones e incluya el trabajo del profesor y del alumno; y la organización de nuevos modelos de formación centrados en el trabajo y el aprendizaje del estudiante.

Este último punto, tema de la presente comunicación, implica innovaciones en las formas tradicionales de enseñar, en consonancia con la construcción del

Scire. $10: 1$ (en.-jun. 2004) 169-183. 
Espacio Europeo de Enseñanza Superior, y la implantación de nuevas metodologías docentes en algunas asignaturas de Documentación. Este proceso de convergencia obliga a redistribuir la carga docente en teoría y práctica, seminarios, tutorías, trabajos de campo, horas de estudio, exámenes, actividades... y, dado que los nuevos curricula se definen en función no sólo de los contenidos, sino también de las capacidades que se adquieren, se requieren métodos de enseñanza que fomenten la competitividad en el mercado de trabajo. Para la Conferencia de Rectores (CRUE, 2001, p. 3):

las universidades ofrecen la posibilidad de encarar la formación orientada a las profesiones dentro de un ambiente académico que garantiza una formación sólida, en la que fundamentos, técnicas, habilidades y aptitudes se adquieren en entornos intelectualmente estimulantes. Así pues, además de propiciar diferencias en los estudios de pre y posgrado de acuerdo con las distintas competencias para el empleo de ambos niveles de estudio, la relevancia social de los estudios dependerá en gran medida de la calidad de la educación recibida, de la diversidad y flexibilidad de programas con múltiples puntos de acceso y salida, del desarrollo de aptitudes y habilidades para la comunicación, la capacidad de jerarquizar la información, y el trabajo en equipo.

La enseñanza magistral, tan útil para la transmisión de contenidos, cuenta todavía con un profundo arraigo la universidad española. Sin embargo, el aprendizaje de capacidades y habilidades necesitan de un componente práctico que, según Corcuera y Pagani (2002), obligará al profesor a un replanteamiento de la organización, contenidos y metodologías aplicables, lo que ha de implantarse progresivamente.

Resumiendo el significado de la calidad en la docencia podemos afirmar que ésta se perfecciona en la efectividad del aprendizaje, es decir, cuando se educa de forma integral: aprendiendo a saber, aprendiendo a saber hacer, aprendiendo a trabajar juntos y aprendiendo a ser, según la UPV VICAA-ICE-GRAD (2001). En nuestra consideración, sin la experiencia previa del docente es imposible un cambio de tal calibre. Es posible normativizar la reorganización de la estructura de los estudios, implantar sistemas de calidad, armonizar títulos y documentos...; pero la adaptación de las metodologías docentes para que el alumno aprenda de forma integrada es patrimonio exclusivo de cada uno de los profesores, y, sin duda, el esfuerzo adicional que supone debe ser incentivado por parte de sus universidades y desde las autoridades educativas.

La Universidad Politécnica de Valencia ha recorrido desde 1988 un largo trayecto en el estímulo de mejoras del sistema de enseñanza-aprendizaje. El Plan de Innovación Educativa (PIE), posteriormente denominado Plan de Innovación Docente (PID), se convoca con una periodicidad anual. El objetivo de 1988 era enfocar la enseñanza hacia las habilidades que debe adquirir un alumno (saber hacer), y más específicamente redistribuir los créditos evitando el peso de la teo- 
ría y fomentando los problemas y laboratorio; implantar sistemas de evaluación continua que contemplasen el dominio de las técnicas, las capacidades de interrelación, la iniciativa del estudiante... En el año 2000, ésta y otras iniciativas de gestión educativa se reúnen en el Proyecto Europa (Una Enseñanza ORientada al APrendizaje), con las siguientes directrices relacionadas directamente con nuevas metodologías docentes (Proyecto, 2000): enfocar las enseñanzas a la consecución del saber hacer del alumno; conseguir, además, que el alumno desarrolle al máximo su capacidad de autoaprendizaje; incidir decididamente en la mejora de los sistemas de evaluación, favoreciendo la evaluación continua y la medida del saber hacer real del alumno; y poner la enseñanza al servicio de la sociedad, orientando la docencia hacia el empleo.

Desde entonces afecta a centros, departamentos y profesorado, y se concreta en cinco programas: Programa de ayuda complementaria a la enseñanza (ACE), Programa de ayuda a la organización docente (ADO), Programa de ayuda a la formación integral del alumno (AFI), Programa de ayuda a la mejora en el aprendizaje (AMA), Programa de ayuda a la mejora de la enseñanza (AME). Este último, AME, favorece las sinergias entre cada centro y los departamentos que imparten docencia en él y apoya las iniciativas encaminadas a (UPV. VICAA-ICE-GRAD, 2001): coordinar mejor los programas de las asignaturas de un plan de estudios (AME1), incentivar la aplicación de nuevos métodos de enseñanza-aprendizaje (AME2), facilitar la implantación de sistemas de evaluación que manejen más información de cada alumno (AME3), y poner en marcha acciones coordinadas para mejorar la cualificación pedagógica del profesorado (AME4).

\section{Objetivos}

La línea en que se inserta nuestro proyecto es AME2-3 y con ella se ha conseguido reconocer en el Plan de Ordenación Docente las actividades del profesorado para imbricar su docencia en un nuevo modelo de aprendizaje. Acogerse a este tipo de incentivos supone para el profesor el reconocimiento de estas actividades por parte de la universidad, tal y como recomienda el MECD (2003) en su documento marco. Unas actividades que hasta ahora se realizaban de manera voluntaria y a partir del curso 2004-2005 se realizarán de forma obligada en la Universidad Politécnica de Valencia.

El objetivo general del proyecto trata de simular una experiencia de ejercicio profesional a través de la "Producción audiovisual para Internet sobre contenidos de Arte Contemporáneo". Durante la convocatoria 2002-2003 varios profesores propusimos al Vicerrectorado de Coordinación Académica y de Alumnado (VICAA) experimentar nuevos métodos docentes y de evaluación, comprometiéndonos en un proyecto común que involucrase la energía de los estudiantes y sus diferentes capacidades. El producto final consistía en la realizacion de docu- 
mentales de 20 minutos aproximadamente sobre arte valenciano con el siguiente formato: 1 minuto para la cabecera y la imagen gráfica de la serie; 3 minutos para la introducción al personaje, presentada por un crítico de arte; 10 minutos para la entrevista, obra, grabación de su estudio, exposición de técnicas de trabajo...; 5 minutos de agenda - promoción de galerías, eventos, flash, espacio critico, debates...-; y 1 minuto para los créditos, esto es, los participantes, incluidos los alumnos colaboradores y los becarios.

Esta producción se erige como una excusa de aprendizaje en la que se integran varios perfiles de estudiantes, dado que el Departamento de Comunicación Audiovisual, Documentación e Historia del Arte (DCADHA) está compuesto por tres áreas diferenciadas cuyas sinergias han facilitado este trabajo común, orientado a la difusión y preservación del patrimonio artístico y documental. Supone por tanto que el alumno se integra en un grupo de trabajo multidisciplinar, en el que es imprescindible la capacidad de trabajar en equipo con otros profesionales. Además, la realización de un producto obliga a que sus prácticas sean "reales", con unos requisitos y plazos que van a condicionar el trabajo del resto de estudiantes. Por último, desarrollan habilidades para documentar un tema en el que no son especialistas: el arte contemporáneo valenciano.

Además de estos objetivos para su capacitación, existen otros transversales al proyecto: conocer nuevas fuentes de información, nuevas maneras de planificar productos informativos y formas de gestionar los derechos de autor y reproducción para contenidos audiovisuales mediante una experiencia práctica; formar a los alumnos en nuevas técnicas de integración de conocimientos a través de un producto audiovisual; conocer los problemas que representa el acceso al documento primario; producir contenidos audiovisuales para la UPV TV y otros; colaborar con la transversalidad de las titulaciones; fomentar la imagen corporativa de las organizaciones - en este caso, de la UPVTV - a través del diseño de una serie estable de productos audiovisuales tales como programas monográficos de autor, de institución, de obra o temáticos; experimentar con la generación de música o la compra de derechos musicales; conocer formas de financiación externas de productos audiovisuales; experimentar otras formas de explotación posterior de los productos, como empaquetar los contenidos no obsoletos en DVD o video streaming; analizar la distribución de contenidos audiovisuales por Internet; y experimentar con nuevos productos para la difusión del arte contemporáneo. Por último, el objetivo docente que planteamos desde un principio fue experimentar con este primer proyecto para establecer una metodología que permitiese sentar las bases de su aplicación en asignaturas y años posteriores. Es decir, encontrar una estructura cerrada de documental que permitiera a los estudiantes aprender el oficio de documentalista (entendido como productor de documentales) para generar una serie infinita de ellos o innovar sobre un producto cerrado.

Scire. $10: 1$ (en.-jun. 2004) 169-183. 


\section{Metodología}

El método para implantar un proyecto de esta naturaleza no puede preverse totalmente a priori, pues son muchas las asignaturas y estudiantes involucrados en él. Se consideraron, no obstante, los siguientes aspectos.

1. Selección de capacidades: Para su consecución se cuenta de partida con las capacidades de cada uno de los perfiles y se ajustaron las asignaturas de las titulaciones que las albergan: Fuentes de información para productos audiovisuales, impartida en la Licenciatura de Documentación por las profesoras Fernanda Peset Mancebo, Antonia Ferrer Sapena (Recursos INformativos); Planificación de productos audiovisuales y gestión de derechos de la información, impartida en la Licenciatura de Documentación por la profesora Nuria Lloret Romero (PSI planificación de sistemas de información); Desarrollo de guiones para productos audiovisuales de arte, impartida en la Licenciatura de Bellas Artes por el prof. José Luis Clemente Marco. (perfil Historia del Arte) (HA Fundamentos Historia del Arte); Adaptación y desarrollo de guiones técnicos para el rodaje de programas de TV e Internet, impartida en la licenciatura de Bellas Arte por el profesor Fernando Canet Centellas (perfil Comunicación Audiovisual, PRODuccion de audiovisuales); y Realización y postproducción de productos audiovisuales para TV e Internet, impartida en la Licenciatura de Bellas Artes por el profesor Adolfo Muñoz García (perfil de Comunicación Audiovisual, GUIones narrativos); y Proyectos, de la Licenciatura en Documentación, impartida por la profesora Luisa Tolosa Robledo (PROYectos).

2. Ajuste del calendario: La complejidad de convertir un proyecto real en una metodología integrada en varios planes de estudio obligó a fijar un calendario acorde con las tareas. Con ello se constató la excesiva rigidez de los horarios para la implantación de estas iniciativas (figura 1).

\begin{tabular}{|c|c|c|c|c|c|c|c|c|}
\hline \multirow[t]{2}{*}{ Id } & \multirow[b]{2}{*}{ (i) } & \multirow[t]{2}{*}{ Nombre de tarea } & \multirow[t]{2}{*}{ Duración } & \multirow[t]{2}{*}{ Comienzo } & \multicolumn{2}{|l|}{2003} & 2004 & 2005 \\
\hline & & & & & \begin{tabular}{|l|l|} 
tri 1 & tri 2 \\
\end{tabular} & \begin{tabular}{|l|l|}
$\operatorname{tri} 3$ & $\operatorname{tri} 4$ \\
\end{tabular} & \begin{tabular}{|l|l|l|l|} 
tri 1 & tri 2 & tri 3 & tri 4 \\
\end{tabular} & \begin{tabular}{|l|l|} 
tri 1 & tri 2 \\
\end{tabular} \\
\hline 1 & & Producción audiovisual para Inter & 521 días & Iun $03 / 02 / 03$ & & & & \\
\hline 2 & 四 & Búsqueda de información & 120 días & Iun 03/02/03 & & & ativos 37 & \\
\hline 3 & 酎 & Diseño Base de Datos & 60 dias & Iun 03/03/03 & & Guiones & tive & \\
\hline 4 & 四 & Informe final & 30 dias & mar 01/06/04 & & & Recurso & informati \\
\hline 5 & 四 & Entrega de documentación & 11 días & Iun 01/09/03 & & & ores 7 & \\
\hline 6 & & Análisis de la documentación & 15 días & mar 16/09/03 & & & icación de Servi & de Infort \\
\hline 7 & 西 & Análisis de la documentación & 15 dias & mar $16 / 09 / 03$ & & & mentos de Hist & del arte 1 \\
\hline 8 & 国 & Elaboración de guiones & 90 días & mié 01/10/03 & & & Fundamentos & listória de \\
\hline 9 & 匞 & Planificación de la producción & 120 días & Iun $06 / 10 / 03$ & & & Planificació & Servicios \\
\hline 10 & 酋 & Entrega de guiones & 15 dias & Iun $22 / 12 / 03$ & & & $F_{1}$ & tória del : \\
\hline 11 & 隁 & Entrega de planning & 15 dias & Iun $22 / 12 / 03$ & & & Planificación de & vicios de \\
\hline 12 & 医 & Producción del documental & 210 dias & Iun $12 / 01 / 04$ & & & & ducción $\mathrm{c}$ \\
\hline 13 & 區 & Montaje del programa & 30 dias & Iun 03/05/04 & & & A & de audio \\
\hline 14 & 匞 & Video Streaming & 66 días & Iun 01/11/04 & & & & Guione: \\
\hline
\end{tabular}

Fig. 1. Planning del proyecto

Scire. $10: 1$ (en.-jun. 2004) 169-183. 
3. Aplicación de nuevas tecnologías: Para la comunicación entre todas las asignaturas y estudiantes se diseñó un sistema de información estructurado a la medida de las fases del proyecto. La herramienta informática, Telearte-Ame3/ AM-AME, diseñada por el profesor Adolfo Muñoz, permite su alimentación por web en http://www.dcadha.upv.es/amame y almacena la información de todo el proyecto, especialmente la recuperada durante la fase de documentación. Un sistema de autorizaciones individuales organizadas en grupos permite administrar e introducir la información estructurada en registros muy simples, diseñados expresamente para los objetivos del proyecto. Dado que para el uso posterior de la información era más relevante saber el género - reseña, bibliografía, crítica, entrevista al autor... - que el soporte, se consignó este en las características del contenido, lo que no es frecuente en bases de datos convencionales de tipo bibliográfico.

4. Adaptación de las asignaturas según la carga de trabajo del estudiante y del profesor: Para llevar a cabo el proyecto había que reorganizar la docencia de las asignaturas según el modelo ECTS, es decir rediseñarlas totalmente para que incluyeran todo el trabajo necesario que un estudiante realiza para aprobar. Así por ejemplo, la primera asignatura Recursos Informativos, optativa semestral, que contaba con 6 créditos - tres teóricos, 1,5 prácticos en aula y 1,5 prácticos en laboratorio- se convertiría en 6 créditos ECTS si el futuro plan de estudios incluyese, como actualmente lo hace, cinco asignaturas por semestre. Esto significa unas 180 horas de trabajo total del estudiante, incluyendo clases teóricas, clases prácticas, seminarios, tutorías, trabajos, horas de estudio, exámenes y otros tipos de evaluaciones. Para el profesor también supone un aumento de horas, especialmente por la cantidad de seguimiento tutorizado que ha de realizar. En el caso de esta asignatura la metodología docente se centra en el aumento del tiempo de prácticas y actividades formativas, orientando todas las capacidades y conocimientos del alumno al apoyo documental del producto que se presenta.

5. Motivación del estudiante: El aprendizaje supone un proceso que se compone de varias fases (figura 2): introducción, desarrollo y consolidación de conocimientos. Nuestra experiencia como docentes llevó a replantear la primera de las fases, frecuentemente olvidada a favor de la segunda y tercera. Durante las primeras sesiones se pusieron en práctica técnicas de motivación del estudiante, explicando detalladamente el proyecto, su alcance... en la primera, visionando otros productos similares en la segunda, e invitando al resto de profesores implicados en el conjunto del proyecto en la tercera (Prof. Muñoz y Clemente).

6. Recursos humanos y materiales: Para un proyecto de estas características se contó con la colaboración de todos los profesores mencionados con anterioridad y tres becarios, dos con perfil de documentalistas y uno con perfil de comunicación audiovisual (Julio Ruiz, Lucas Huesca y Alejandro Portaz). Los

Scire. $10: 1$ (en.-jun. 2004) 169-183. 


\section{PROCESO}

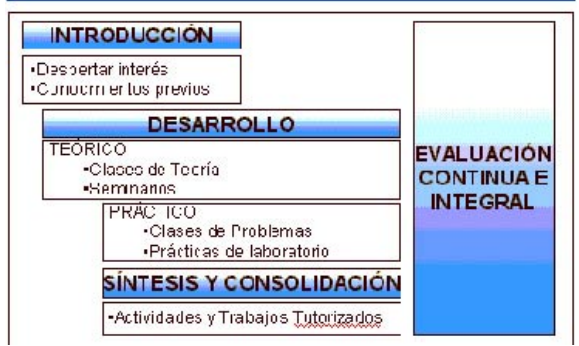

Fig. 2. Proceso de aprendizaje (UPV. VICAA-ICE-GRAD, 2001)

recursos materiales utilizados fueron un servidor en Internet, administrado por un profesor, y un escáner, un ordenador portátil y una grabadora de CD's a disposición de todo el personal.

\section{Resultados}

Los resultados del proyecto son todavía parciales, aunque consideramos que algunos pueden extrapolarse al resto de asignaturas. Por otra parte, considerando que cada profesor es el único experto que puede decidir cuál es la metodología apropiada para su asignatura, presentamos la que se refiere a Recursos Informativos, cuyo diseño conforme a las innovaciones metodológicas descritas anteriormente ya está concluido. Esta asignatura, cuya carga docente ya se ha detallado, es una optativa de segundo ciclo que este año ha alcanzado su máximo número de alumnos (figura 3), si bien no se debe a la nueva metodología implantada, ya que los estudiantes fueron informados de ello una vez matriculados.

Hasta ahora, los tres créditos teóricos se ofertaban también de manera no presencial y las prácticas en laboratorio eran diseñadas en consonancia con los contenidos teóricos e ilustraban individualmente cada una de las lecciones. En lo referente a la evaluación, además de la entrega obligatoria de las prácticas, la asignatura se superaba mediante trabajos. Ante los resultados de años precedentes, progresivamente se aumentó la carga de tutorización de trabajos mediante el establecimiento de plazos de entrega de temas, bibliografía y guiones para su aprobación por el profesor. Por ello, la comunicación con los alumnos se produce tanto durante las clases teóricas y prácticas, como por correo electrónico y avisos colectivos a alumnos de forma electrónica.

Este método, si bien contaba con índices aceptables de aprobados, no era suficiente para motivar a los alumnos, pues teoría y prácticas eran diseñadas de 


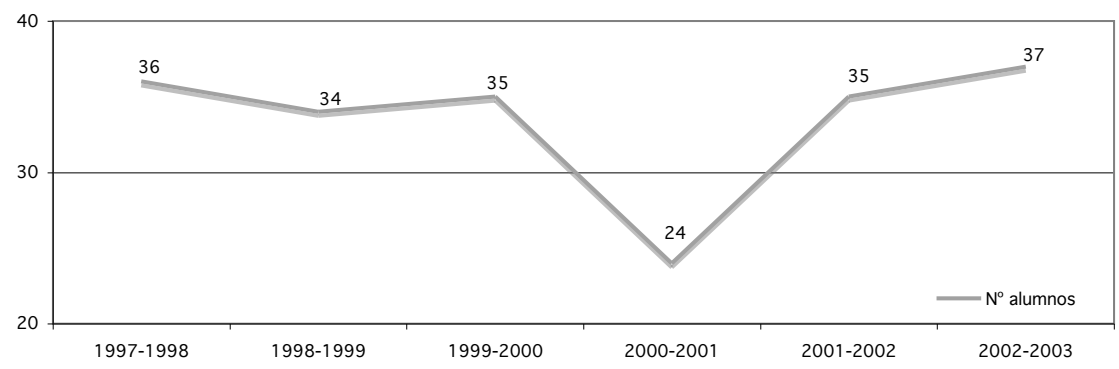

Fig. 3. Evolución de la matrícula

forma “artificial”. El docente diseña generalmente los contenidos y sus prácticas sin un objetivo conjunto, sino orientadas al conocimiento abstracto de aspectos concretos de la documentación. Por ello, existía una disfunción entre la materia impartida, de carácter general, y la evaluación, ya que los trabajos abordaban profundamente una materia determinada, dado que se trata de un segundo ciclo. El perfil de los alumnos estaba compuesto por diplomados en Biblioteconomía y Documentación, formados en fuentes de información bibliográfica, y con habilidades para el manejo de bases de datos de Documentación Científica e Internet - motores de búsqueda generalistas e información textual-. El nuevo método, descrito en parte, ha supuesto una adaptación de los contenidos teóricos al arte contemporáneo valenciano y la inclusión de nuevos contenidos afines a la asignatura: por ejemplo, el funcionamiento del mercado del arte y sus fuentes de información. Por su parte, las prácticas se han orientado totalmente a la consecución de la documentación de los temas propuestos, así como a la búsqueda de información hipermedia no convencional.

Durante el curso fueron documentados los temas establecidos por el profesor José Luis Clemente, referentes a personas -artistas y directores de organizaciones- e instituciones; en concreto los artistas Carmen Calvo, Andreu Alfaro, Miquel Navarro, Equipo Límite, Mira Bernabeu y Daniel G. Andujar; los centros de exposición Instituto Valencia de Arte Moderno (IVAM), Espai d'Art Contemporani de Castelló (EACC), La Gallera, y las galerías Visor, Tomas March, Espai Lucas; y agentes como los directores del IVAM, EACC y Galerías (Tomás March, Pep Benlloch, La Nave) y Ciutadans per una Cultura democràtica i Participativa.

Cada grupo de alumnos debía identificar fuentes de información para arte contemporáneo, y se insistió en la recopilación de material que no fuera exclusivamente textual, dado que el producto final, un documental, se compone básicamente de información audiovisual. La programación de la asignatura se

Scire. $10: 1$ (en.-jun. 2004) 169-183. 


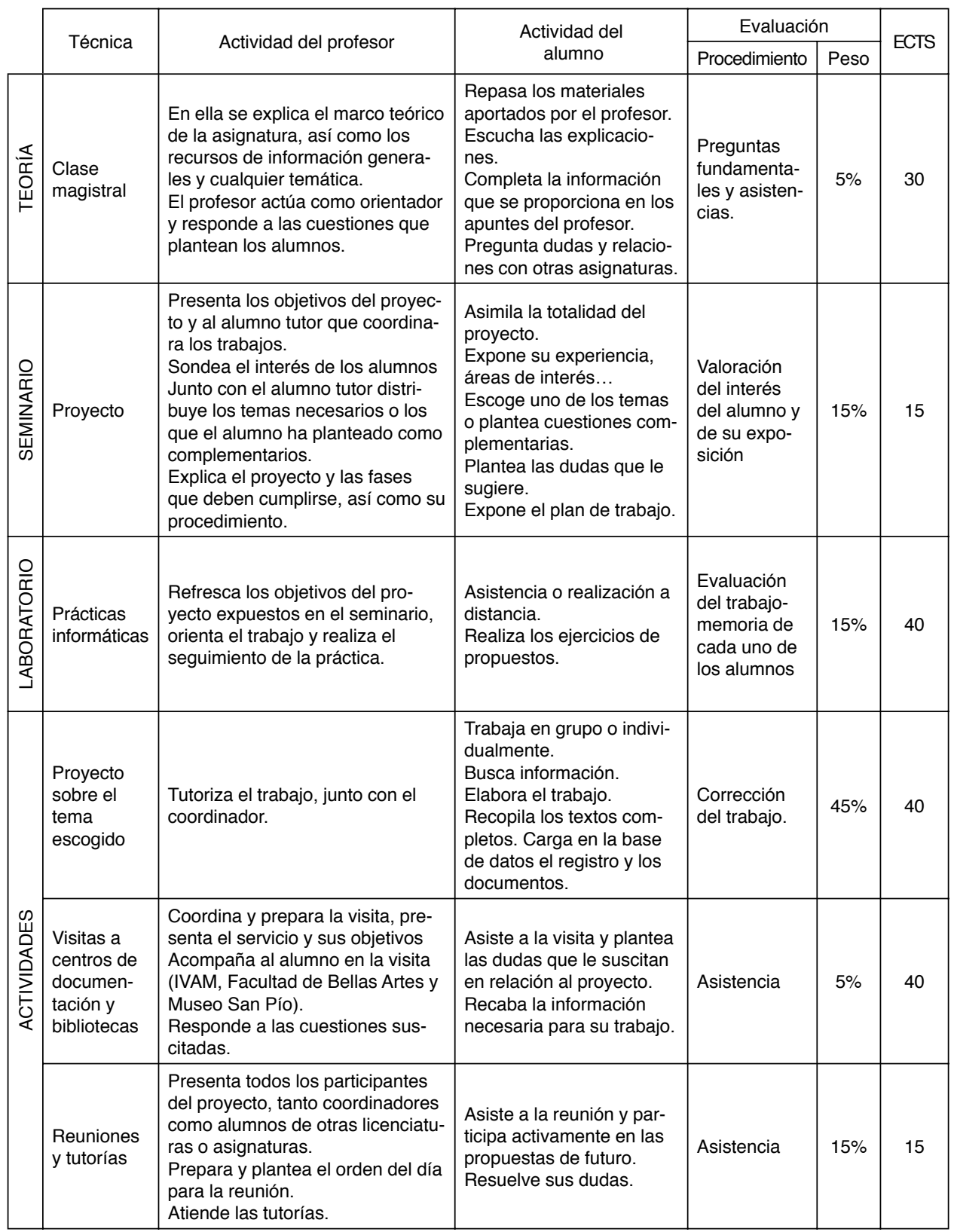

Tabla I. Programación de la asignatura

Scire. 10: 1 (en.-jun. 2004) 169-183. 
muestra en la tabla I en la página anterior, y cabe destacar que teoría, seminario, laboratorio y actividades están totalmente orientados a la realización del producto final. Sin querer extendernos en el desarrollo de los contenidos e incidencias del proyecto resulta significativo que el sistema de comunicación ha aumentado cualitativamente, con mayor número de consultas presenciales y por correo a iniciativa del alumno; así también la asistencia a las prácticas ha sido espectacular en comparación con otros años, teniendo en cuenta su horario, viernes de 19 a 21 horas; por último, para un proyecto tan complejo fueron necesarias siete reuniones de control entre profesores y becarios que se documentaron y difundieron puntualmente. Los resultados obtenidos por los estudiantes, mostrados en el gráfico, manifiestan las dificultades que un documentalista free lance o un investigador pueden encontrar a la hora de documentar temas actuales.

En términos generales los resultados del proyecto han sido satisfactorios y los estudiantes han tenido que realizar un ciclo completo de documentación: identificación de recursos, consulta de bases de datos referenciales, interrogación de catálogos de bibliotecas (con monografías y títulos de revista), obtención del material, digitalización del ítem e inserción del registro y el documento completo en la base de datos. Los alumnos han realizado un gran esfuerzo por realizar las prácticas y desean involucrarse en su seguimiento cursando la asignatura de Planificación de Servicios de Información y ayudando a los estudiantes de historia del arte en la redacción de los guiones con la información que han recuperado.

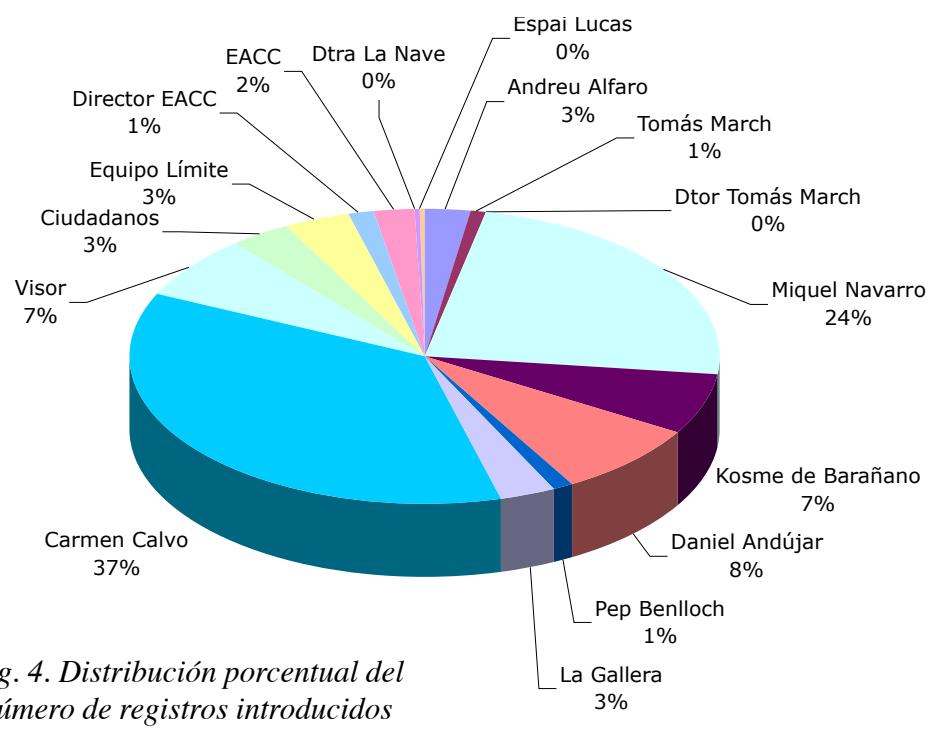

Scire. $10: 1$ (en.-jun. 2004) 169-183. 


\section{Conclusiones}

Las conclusiones que se desprenden la implantación de esta nueva metodología docente han sido aportadas por profesores, becarios y estudiantes.

El primer grupo, los profesores, estimamos a nivel general que el interés por la asignatura ha sido mayor este año, aunque también lo han sido las dificultades de coordinación. Entre múltiples aspectos cabe destacar el acercamiento mayor de esta metodología a la realidad del mercado laboral, en el que, además, se trabaja en grupos de perfiles profesionales heterogéneos. Asimismo, en los grupos de trabajo se han detectado carencias en habilidades básicas - por ejemplo para escanear imágenes y retocarlas - y muy distintos niveles de formación en los alumnos. En este sentido, redactar un informe final de resultados les ha obligado a seguir un método que les permitiese sintetizar su esfuerzo, si bien su evaluación ha reflejado que mucho del trabajo realizado no se encuentra detallado en las memorias. Por el contrario, un grupo ha diseñado, además, un sistema hipermedia e hipertexto para la presentación de su trabajo, lo que demuestra su motivación. Por otra parte, el estudiante se ejercita en la resolución de problemas informativos para un objetivo concreto, con unos plazos y usuarios que van a evaluar inmediatamente el resultado de su trabajo. De hecho, el diseño de la base de datos en función de su uso final ha permitido que los alumnos sean conscientes en todo momento del objetivo del trabajo. Los temas escogidos obligan al estudiante a distinguir las diferentes clases de información, como son la de personas y organizaciones, la información histórica frente a la actual, la información textual frente a la sonora, la imagen fija y la imagen en movimiento... Estas cuestiones, aprendidas en teoría, no las habían interiorizado hasta enfrentarse con los problemas que derivan de su recuperación y almacenamiento. En resumen, extractamos un pasaje revelador (Medina Landete y otros, 2003) de cómo esta nueva metodología ha contribuido a aumentar la confianza del estudiante en sus capacidades, validando el esfuerzo de todos los profesores que contribuimos a su proceso de aprendizaje durante años:

Este trabajo nos ha servido para darnos cuenta que, aún no siendo conocedores del tema, teniendo una buena base y conocimiento de los recursos de información que tenemos a nuestro alcance, es posible localizar todo tipo de información sobre cualquier temática, algo que, al principio de la asignatura, creíamos sería un serio problema para el desarrollo del trabajo.

El segundo grupo, los becarios, han aportado conclusiones relacionadas con la coordinación del proyecto, los medios, la planificación y sus posibles soluciones. Por último, al tercer grupo, alumnos, se les ha solicitado en la memoria de resultados que evalúen la experiencia de forma abierta y que respondieran a la siguiente pregunta: “¿Crees que vale la pena implantar nuevas metodologías docentes en la licenciatura de Documentación - UPV?” De las diecisiete respues- 
tas, tan sólo una persona ha contestado negativamente (6\%). De hecho, resulta alentador que con respecto a esta nueva metodología uno de los grupos reseñe que: "Vale la pena un nuevo sistema que nos enseñe más la realidad de fuera y no tanto estudio teórico" (Ferreras Feliz y otros, 2003). Entre las conclusiones que apuntan, encontramos varias dignas de destacar. Primeramente, el alumno, como cualquier profesional en ejercicio, constata que antes de documentar cualquier tema debe conocerlo, con lo que existe una tarea previa de "autoformación". En este sentido, una de las mayores dificultades que detectan es la evaluación de recursos para el objetivo que se les planteaba, generándoles en unos casos un reto y, en otros, sensación de inseguridad. En todo caso, esta toma de conciencia sobre la utilidad directa de su trabajo otorga mayor valor añadido a su esfuerzo. También reparan en las lagunas que subsisten en las fuentes de información a su alcance, tanto en las colecciones documentales de la ciudad de Valencia, como en la red. Con respecto al primer asunto, contactan con profesionales en activo y destacan su profesionalidad; mientras que en el segundo detectan que puede ser un nicho de trabajo, que además resulta de su interés. En este sentido, constatan que no pueden acudir indiscriminadamente a cualquier recurso de información, y, por tanto, es imprescindible conocer las instituciones documentales, pero también a los propios generadores de los documentos (artistas) u organizaciones documentadas, ya que normalmente guardan la información referente a ellos mismos. Gracias a ello identifican recursos de información "escondidos", invisibles a las grandes bases de datos de datos que generalmente están orientadas a la información bibliográfica, cuya utilidad es la investigación científica. Ya al término de su trabajo, comprueban que para hacer un uso eficiente del tiempo y de su trabajo han de adoptar estrategias y apuntan que "si comenzáramos de nuevo el trabajo, estableceríamos otro método de trabajo" (Escribano Alcaide y otros, 2003). Finalmente, valoran positivamente la identificación y manejo de nuevos tipos documentales, algunos muy específicos del mundo del arte - por ejemplo, catálogos de exposiciones - y otros generalistas - como los fondos hemerográficos locales - que no están sistematizados en ninguna base de datos. De ambos, además, se plantean la extracción de partes de información - por ejemplo fotos-, y esto les obliga a reflexionar cómo citarlas, introducirlas en una base de datos... integrando conocimientos que provienen de otras asignaturas.

En definitiva, consideramos que la convergencia al nuevo espacio de educación superior europeo demanda un gran esfuerzo a los docentes, ya que es posible legislar sobre organización, procedimientos y estructuras generales de los estudios, pero es cada profesor en su propia disciplina quien ha de encontrar métodos propios para desarrollar la docencia de calidad. Para Docampo (2001, p. 10):

el quicio de la reforma no está en la acomodación de los contenidos de los planes de estudio a la nueva estructura sino en un cambio de paradigma: pasar de una educa-

Scire. $10: 1$ (en.-jun. 2004) 169-183. 
ción centrada en la enseñanza (en el profesor) a otra centrada en el aprendizaje (en el alumno). Esta cultura del aprendizaje debe llevarnos a una revisión profunda de nuestros esquemas de evaluación, actualmente estructurados en torno a la dualidad aprobado/suspenso, con el fin de reflejar adecuadamente en las calificaciones el esfuerzo efectuado por los estudiantes.

Deberíamos por tanto evitar la adopción de cambios cosméticos, que simplemente acomodan contenidos sin modificar mentalidades; si el estudiante debe pasar a ser el centro del proceso de aprendizaje, las aulas universitarias deben ser por tanto lugares a los que se va a aprender (no a enseñar). No hay que olvidar que la ciudadanía espera recibir de las universidades profesionales que hayan desarrollado aptitudes y habilidades para ser útiles a la sociedad.

En este sentido, cabe reseñar que cada uno de los profesores implicados en este proyecto se ha asignado un rol voluntariamente en función de sus capacidades, tal como ocurre en todo trabajo en equipo, aun sin ser periodo de su docencia. Con ello se alcanza el objetivo de participar en un proyecto común, con perfiles interdisciplinares, algo muy difícil de conseguir con la antigua forma de impartir las asignaturas.

\section{Recomendaciones}

La implantación de nuevas metodologías docentes en el ámbito de cualquier disciplina recomienda la modificación profunda de los planes de estudio atendiendo a estas nuevas formas de enseñanza. Pero también exige una mayor implicación de la organización en cuanto a reconocimiento de carga docente y medios al alcance de profesorado y estudiantes. En este sentido, parece que el legislador contempla estas exigencias, de manera que es de suponer que estas iniciativas serán fomentadas y apoyadas con recursos. Por último, en la línea de la Reunión de decanos y responsables de Documentación de 22 de abril (2003), consideramos recomendables las reflexiones de esta comunicación para tres de los apartados que allí se plantean, a saber la definición del perfil profesional de los titulados en el segundo nivel, de los objetivos generales y las competencias y destrezas generales de la titulación, y de los contenidos fundamentales y optativas. Nunca olvidemos que la fecha límite para la creación de un espacio europeo de enseñanza está cercana: 2010.

\section{Referencias}

Bayod, José Manuel; et al. (2003). Documento de trabajo sobre la integración de los estudios españoles de matemáticas en el espacio europeo de enseñanza superior. Sevilla: Universidad de Sevilla, marzo 2003. URL: <http://www.matematicas.us.es/Bolonia/ documento_final.pdf>. 
Comunicado de Praga (2003). Towards the European Higher Education Area: Communiqué of the meeting of European Ministers in charge of Higher Education in Prague on May 19th 2001. [Bruselas]: Unión Europea, [2001]. URL: <http://www.crue.org/ comcumbrepraga.htm>.

CORCUERA, Fidel; PAGANI, Raffaella. Los créditos ECTS. Baiona-Vigo, 4 noviembre 2002. [Madrid]: CRUE, [2003]. URL: <http://www.crue.org/ppt/CreditosECTS(Corcuera-Pagani).ppt>.

CRUE (2001). La Declaración de Bolonia y su repercusión en la estructura de las titulaciones en España: Propuesta de Posición de la CRUE: Aprobado en la reunión de la CASUE de 26 de octubre de 2001. Madrid: CRUE, 2001. URL: <http:// www.unizar.es/eees/documentos/CRUE\%20Posicion\%20CASUE\%20261001.pdf>.

Declaración de Bolonia (2003). The European Higher Education Area: Joint declaration of the European Ministers of Education: Convened in Bologna on the 19th of June 1999. [Bruselas]: Unión Europea, [1999]. URL: <http://www.crue.org/ decbolognaingles.htm>.

Declaración de la Sorbona (1998). Joint declaration on harmonisation of the architecture of the European higher education system, by the four Ministers in charge for France, Germany, Italy and the United Kingdom. Paris, the Sorbonne, May 25 1998. URL: $<$ http://www.crue.org/sorbo-in.htm>.

Docampo, Domingo (2001). La Declaración de Bolonia y su repercusión en la estructura de las titulaciones en España_Navarra-Universidad de Navarra, 2001. URL: <http:// www.unavarra.es/directo/fcee/Europa/Docampo.pdfp.

Escribano Alcaide, Raúl; Martí Martí, Federico; López Camps, Genoveva (2003). Informe de resultados. Valencia: Universidad Politécnica, 2003. Inédito.

Ferreras Feliz, Vanesa; Nadal Sanchís, Maria; Seguí Morant, David; Serra Alegre, César (2003). Informe de resultados. Valencia: Universidad Politécnica, 2003. Inédito.

Ley Orgánica 6/2001, de 21 de diciembre, de Universidades. Madrid: MECD, [2002]. URL: <http://www.univ.mecd.es/sgrju/disposiciones/leyes/leyo6-01.html>.

Medina Landete, José Miguel; Juliá Alcañiz, Mónica; Sanz Albillo, Carmen I.; Sangrós García, Teresa. Informe de resultados. Valencia: Universidad Politécnica, 2003. Inédito.

Ministerio de educación, cultura y deporte. La integración del sistema universitario español en el espacio europeo de enseñanza superior: documento-marco. Madrid: Ministerio de educación, cultura y deporte, febrero 2003. URL: <http://www.univ.mecd.es/ internacional/convergencia_europea.html.

Proyecto Europa. 2. Objetivos. Valencia: Universidad Politécnica de Valencia, 2000. URL: $<$ http://www.upv.es/europa/intro.htm>.

Reunión de decanos y responsables de titulaciones de Biblioteconomía y Documentación. Barcelona, 22 de abril de 2003: Estructura de las titulaciones de ByD en el nuevo espacio europeo. Barcelona: Universidad de Barcelona, 2003. Inédito.

UPV (2001). VICAA-ICE-GRAD. Nuevas metodologías docentes y de evaluación. Valencia: Universidad Politécnica, 2001. URL: <http://www.upv.es/upl/ U0029606.pps>.

Scire. $10: 1$ (en.-jun. 2004) 169-183. 